\title{
Review
}

\section{Cellular trafficking of nicotinic acetylcholine receptors}

\author{
Paul A ST JOHN* \\ Department of Cell Biology and Anatomy, University of Arizona College of Medicine, Tucson, AZ 85724, USA
}

Nicotinic acetylcholine receptors (nAChRs) play critical roles throughout the body. Precise regulation of the cellular location and availability of nAChRs on neurons and target cells is critical to their proper function. Dynamic, post-translational regulation of nAChRs, particularly control of their movements among the different compartments of cells, is an important aspect of that regulation. A combination of new information and new techniques has the study of nAChR trafficking poised for new breakthroughs.

Keywords: membrane traffic; protein traffic; biosynthesis; endocytosis; endoplasmic reticulum-associated degradation Acta Pharmacologica Sinica (2009) 30: 656-662; doi: 10.1038/aps.2009.76

\section{Introduction}

Nicotinic acetylcholine receptors (nAChRs) mediate synaptic transmission in the CNS, in autonomic ganglia, and at neuromuscular junctions and other peripheral synapses. The functional properties of these synapses differ, but in each case, properly functional signaling requires cellular control of the number, type, and location of nAChRs. Trafficking of nAChRs - the movement of nAChRs between compartments of a cell, including the cell's biosynthetic and degradative compartments - is an important aspect of such control. Research on the trafficking of nAChRs has been increasingly well informed in recent years by knowledge of trafficking events and mechanisms in other cell types and trafficking of other types of receptors. Now powerful imaging methods and new findings concerning molecular mechanisms are generating excitement for a deeper understanding of this topic.

The trafficking of G-protein-coupled receptors has received extensive study, may be clinically relevant, and is increasingly well understood. Ionotropic receptors have now been shown to undergo tightly regulated trafficking, as well. The regulated trafficking of ionotropic glutamate receptors, for example, appears to play an important role in forms of synaptic plasticity that are thought to be important in learning and memory ${ }^{[1-3]}$.

The trafficking of nAChRs may be regulated in several

* Correspondence to to Prof Paul A ST JOHN.

E-mail: stjohn@u.arizona.edu.

Received 2009-03-02 Accepted 2009-04-28 ways, but two particular perturbations have been especially well studied and exert their effects at least in part by altering the trafficking of nAChRs: 1 ) denervation changes the total number, the distribution, and the turnover rate of nAChRs in skeletal muscle; 2) prolonged exposure to nicotine increases the total number of nAChRs in neurons. Several of the studies reviewed here addressed the mechanisms by which these treatments alter $\mathrm{nAChR}$ trafficking. Other authors in this special issue will address other aspects of the effects of nicotine on nAChRs.

This review will begin with trafficking events and mechanisms in the biosynthetic pathway, and then will turn to those in the endocytic/recycling/degradative pathway. I will not address the clustering of $\mathrm{nAChRs}$ at synapses, which has been reviewed elsewhere ${ }^{[4-6]}$.

\section{Trafficking of nAChRs in the biosynthetic pathway}

The nAChRs of skeletal muscle have been intensely studied for decades. Regulation of the number and location of nAChRs at neuromuscular junctions has been a special focus of many of these studies. Under normal conditions, the overall number and location of nAChRs at neuromuscular junctions changes fairly little over time, as one might expect from considering the role of these synapses in the body. The constancy in the overall number of nAChRs requires that the rate of synthesis and delivery to the cell surface of new nAChRs and the rate of turnover and degradation of old $\mathrm{nAChRs}$ be kept in balance. How that balance is achieved and maintained in normal muscle cells is not fully under- 
stood, but it is commonly thought that the negative feedback between the level of nAChR-dependent activity in a cell and the level of transcription of the genes for $\mathrm{nAChR}$ subunits in that cell plays an important role (see below).

Assembly of new $\mathrm{nAChR}$ subunits into whole receptors soon after their translation in the endoplasmic reticulum
(ER) is a key regulatory step in the trafficking of nAChRs to the plasma membrane (Figure 1). Under normal conditions, only subunits that have been assembled into complete pentameric receptors are transported to the cell surface, while unassembled and incompletely assembled subunits are retained in the ER and ultimately degraded through the
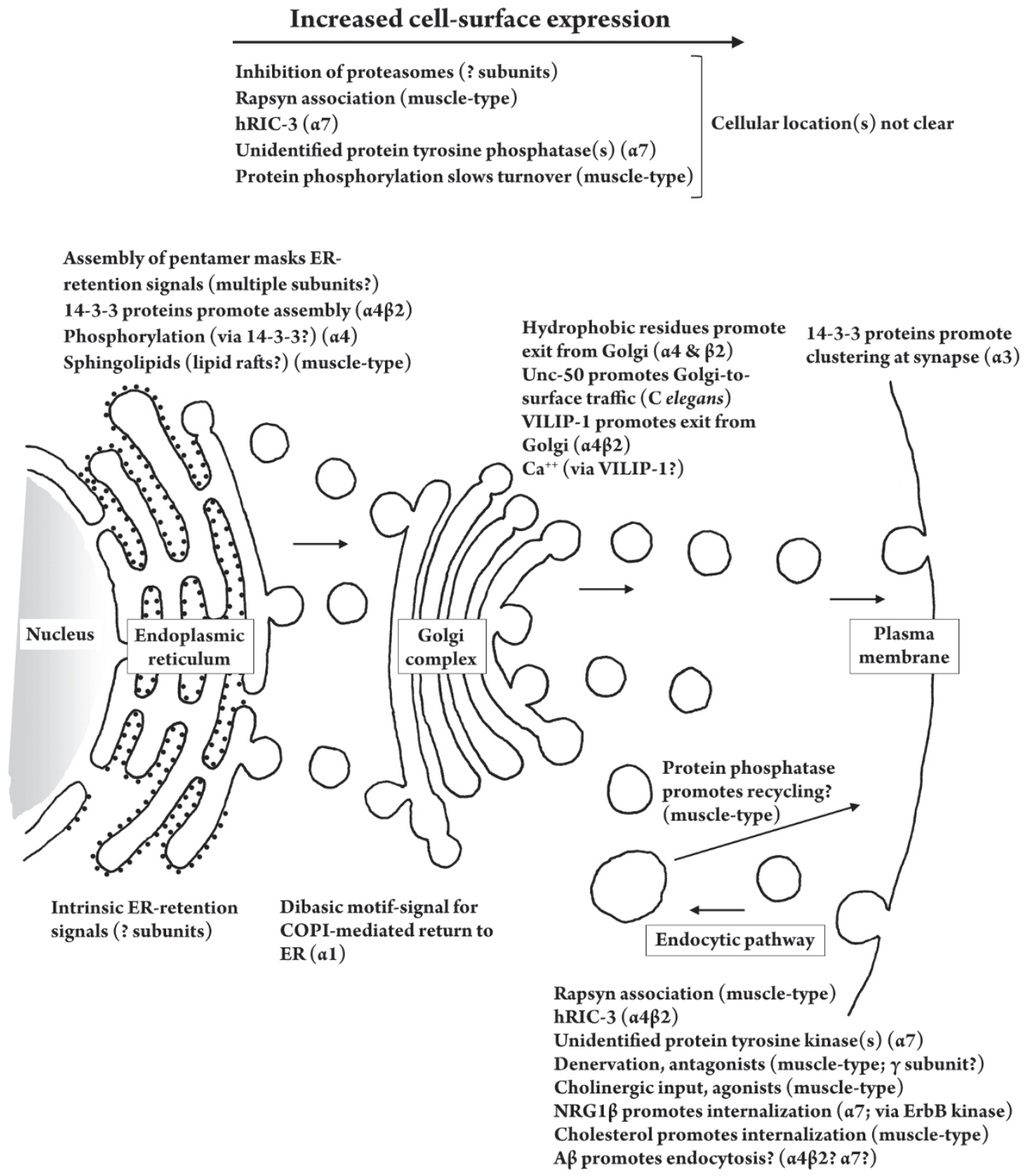

Decreased cell-surface expression

Figure 1. Factors that affect the trafficking of nAChRs. This figure is a summary of factors discussed in the text that affect the cellular trafficking of nAChRs. Factors listed in the upper portion of the figure tend to increase the cell-surface expression of nAChRs, while those listed in the lower portion of the figure tend to decrease surface expression. Factors are located in the drawing near the organelle in which they appear to act, and the nAChR subunit or subunits affected are listed in parentheses, in cases in which that information is available. 
process of ER-associated degradation (ERAD $)^{[7]}$. How is the release of new receptors from the ER controlled? Structural determinants within $\mathrm{nAChR}$ subunits themselves play an important role. Studies by Wang et al ${ }^{[8]}$ showed that a motif within a conserved transmembrane domain of nAChR subunits serves as a molecular determinant for this sorting. Mutation of this motif allowed unassembled nAChR subunits to be transported to the cell surface, and insertion of this motif caused retention in the ER of proteins that normally are transported to the cell surface. Assembly of native $\mathrm{nAChR}$ subunits into pentameric receptors appears to bury this motif and thereby allow the efficient and selective delivery of assembled nAChRs to the cell surface. Keller et al ${ }^{[9]}$ found that mutating a pair of basic amino acids located in the large cytoplasmic loop of the $\alpha 1$ (muscle-type) nAChR subunit diminished the normal association of $\alpha 1$ subunits with COPI, a protein complex involved in transporting proteins back from the Golgi and retaining them in the ER, and allowed trafficking of unassembled subunits from the ER to the Golgi in transfected HEK293 cells. The authors suggested that the ER-retention signals they identified become masked during normal assembly of nAChRs in the ER, thus allowing the new receptors to proceed along the biosynthetic pathway. Because mutant $\alpha 1$ subunits that escaped the ER accumulated in the Golgi in their experiments, the authors concluded that there is a second check-point, and presumably a second set of molecular tags, that controls delivery of new nAChRs to the cell surface. In a study of trafficking determinants within the subunits of neuronal nAChRs, Ren et $a l^{[10]}$ identified specific hydrophobic residues in the cytoplasmic loops of both $\alpha 4$ and $\beta 2$ subunits that are required for surface expression of $\alpha 4 \beta 2$-nAChRs. These structural determinants appear to function downstream of receptor assembly, since mutating them did not alter the binding of epibatidine, but did prevent surface expression.

Additional studies concerning the role of ERAD in the trafficking of nAChRs have shown that inhibition of proteasome activity increases the number of nAChRs on the surface of cultured myotubes, due to increased assembly of $\mathrm{nAChR}$ subunits in the $\mathrm{ER}^{[11]}$. The ubiquitin-proteasome system (UPS) can affect the trafficking and surface expression of neuronal nAChRs, as well.

In a recent review of the effects of nicotine on $\alpha 4 \beta 2$ nAChRs, Corringer et al ${ }^{[12]}$ hypothesized that nicotine acts as a "maturational enhancer" that "works through a yet unknown endogenous substance" to promote receptor expression. Recently, Rezvani et al ${ }^{[13]}$ found that a 24-hour exposure to nicotine reduced proteasomal activity and increased the accumulation of ubiquitinated $\alpha 7-\mathrm{nAChR}$ sub- units, although it was not clear in what cellular compartment the ubiquitinated receptors accumulated. This effect on ubiquitination of nAChRs may be a critical part of the mechanism by which prolonged exposure to nicotine up-regulates the total number of nAChRs. The sustained exposure to nicotine also increased the levels of metabotropic and ionotropic glutamate receptor subunits and glutamate receptorrelated proteins ${ }^{[13]}$. The authors suggest that the UPS might participate in nicotine-dependent synaptic plasticity. How specific such effects would be is not clear, in light of the effects on receptors for other neurotransmitters, but perhaps the UPS also upregulates the expression of $\alpha 4 \beta 2-n A C h R s$ and represents the "unknown substance" hypothesized by Corringer et $a l^{[12]}$.

Several non-receptor proteins have been implicated as possibly regulating the trafficking of muscle-type nAChRs in the biosynthetic pathway. Work by Marchand et al ${ }^{[14]}$ suggested that rapsyn, a $43-\mathrm{kD}$ protein originally identified by its association with $\mathrm{nAChRs}$ at mature neuromuscular junctions ${ }^{[15]}$, can associate with muscle-type nAChRs in a cell's biosynthetic pathway and serve as a chaperone to target the $\mathrm{nAChR}$ to the plasma membrane. In contrast, however, Han et $a l^{[16]}$ found that overexpression of rapsyn reduced the fraction of nAChRs on the cell surface and caused accumulation of nAChRs inside the cell. Additional studies to learn more about the intracellular site(s) of action of rapsyn for trafficking effects might help to resolve the apparent discrepancy between these studies.

In the nematode $C$ elegans, the protein Unc-50, which localizes to the Golgi apparatus, is required for the proper delivery of certain types of nAChRs to the cell surface ${ }^{[17]}$. It will be interesting to learn exactly how Unc- 50 interacts with the nAChRs in that system, and because Unc-50 is evolutionarily conserved ${ }^{[17]}$, it will be intriguing to learn whether vertebrate homologues play a similar role in the trafficking of vertebrate nAChRs.

The neuronal protein VILIP-1 has been implicated in regulating the surface delivery of several types of neurotransmitter receptors ${ }^{[18,19]}$. VILIP-1 increases the surface expression of $\alpha 4 \beta 2$-nAChRs, both in oocytes ${ }^{[18]}$ and in hippocampal neurons ${ }^{[20]}$, possibly by functioning in the biosynthetic pathway to promote exit from the trans-Golgi network and resulting constitutive exocytosis. The authors hypothesize that VILIP-1 is activated by an increase in intracellular $\mathrm{Ca}^{++}{ }^{[20]}$, a mechanism that could tie the control of surface expression to the level and/or pattern of neuronal activity. Work by Nashmi et al ${ }^{[21]}$ suggested that nicotine promotes the assembly of $\alpha 4 \beta 2$-nAChRs via an unidentified $\mathrm{Ca}^{++}$-stimulated pathway. Future studies may reveal whether VILIP-1 
is involved in the effect of prolonged exposure to nicotine.

The "14-3-3" proteins have been shown to allow or promote forward trafficking of multimeric plasma-membrane proteins by masking or overriding COPI recognition signals that otherwise would cause the proteins to be retained in the $\mathrm{ER}^{[22]}$. Bermudez et al ${ }^{[23]}$ found that phosphorylation of a4-nAChR subunits at a PKA consensus sequence promotes binding of 14-3-3 proteins to the a 4 subunits in the ER and their stabilization there. This in turn appears to promote the assembly of complete $\alpha 4 \beta 2$-nAChRs, which then can be released from the ER and traffic to the plasma membrane. It is not known whether changes in 14-3-3 proteins could dynamically regulate the surface expression of $\alpha 4 \beta 2$ nAChRs in response to physiological or environmental cues, or whether they influence the surface expression of a7-containing or other subtypes of nAChRs. It is interesting to note that 14-3-3 proteins recently have been implicated in the clustering of $\alpha 3$-containing $\mathrm{nAChRs}$ at synapses on the surfaces of ganglionic neurons ${ }^{[24]}$.

The vertebrate homologue of a protein called RIC-3, which was originally identified as being required for maturation of nAChRs in the nematode $C$ elegans, has interesting effects on $\mathrm{nAChR}$ trafficking, in that it appears to exert opposite effects on $\alpha 4 \beta 2$ - and $\alpha 7-n A C h R s^{[25]}$. hRIC-3 enhances the surface expression of $\alpha 7$-nAChRs, but dramatically inhibits that of $\alpha 4 \beta 2$-nAChRs, apparently by hindering their export from the biosynthetic pathway to the plasma membrane ${ }^{[25]}$. Future studies should provide more information concerning the exact mechanism of interaction between RIC-3 proteins and nAChR subunits, including the factors that account for apparently opposite effects on different subtypes of nAChRs.

Protein phosphorylation appears to modulate the trafficking of nAChRs. Cho et al ${ }^{[26]}$ found that an inhibitor of protein tyrosine kinases potentiated $\alpha 7$-nAChR-mediated responses to ACh in oocytes and in cultured hippocampal neurons, while a protein tyrosine phosphatase inhibitor caused depression of such responses. Kinase inhibition caused an increase in the number of $\alpha 7-\mathrm{nAChR}$ subunits, with no change in channel open probablility. Mutation of the cytoplasmic tyrosines of the $\alpha 7$ subunits did not prevent the potentiation by the kinase inhibitor, suggesting that the effect on trafficking could be exerted through phosphorylation of some other protein or proteins. The authors also provided evidence that SNARE proteins, well-known for their role in trafficking in other cell types, play some role in the changes they observed.

Membrane cholesterol and other lipids also have been found to play a role in the aggregation and trafficking of muscle-type nAChRs. In CHO-K1/A5 cells, which express muscle-type nAChRs, impairing the synthesis of sphingolipids decreases the surface expression of nAChRs, apparently by affecting the biosynthetic pathway ${ }^{[27]}$ : when sphingolipids are depleted, newly synthesized but unassembled nAChRs accumulate in the endoplasmic reticulum (ER), and fail to be transported to the cell surface. The latter effect, at least, might in part be due to an effect on so-called lipid rafts in cells, as Marchand et al ${ }^{[14]}$ found evidence that nAChRs and the receptor-associated protein rapsyn are transported through the biosynthetic pathway in association with lipid rafts, which suggests that disruption of the rafts could interfere with the delivery of new nAChRs to the cell surface. It will be interesting to learn more about the molecular interactions that underlie the effects of lipid perturbations on the trafficking of nAChRs.

Several studies have shown that prolonged exposure to nicotine can increase, or up-regulate, the number of nAChRs, in the brains of smokers, in laboratory animals, and in cultured cells ${ }^{[28]}$. Questions about the subunit-selectivity of this effect and the contribution of transcriptional regulation to it will be addressed by other authors in this issue. However, at least part of the effect of chronic nicotine on nAChRs appears to be post-translational, and to involve trafficking effects, primarily or exclusively in the biosynthetic pathway. Darsow et a ${ }^{[29]}$ found that the nicotine-induced increase in the number of $\alpha 4 \beta 2$-nAChRs in HEK cells was due to an increase in the transport of $n A C h R s$ through the biosynthetic/secretory pathway, with no change in the rate of internalization or trafficking in the endocytic pathway. This contradicts the conclusions of Vallejo et al ${ }^{[30]}$ that nicotine stabilizes a high-affinity binding state of $\alpha 4 \beta 2$-nAChRs, thus accounting for increased binding of ligands, but does not alter the assembly, trafficking, turnover, or total number of nAChRs on the cell surface.

\section{Trafficking in the endocytic/recycling/degradative pathway}

As mentioned above, denervation of skeletal muscle increases the number of nAChRs, which reflects a large increase in transcription of genes for $\mathrm{nAChR}$ subunits ${ }^{[31]}$. Denervation also alters a trafficking property of $\mathrm{nAChRs,}$ namely, the turnover rate of receptors on the cell surface. For example, Bevan and Steinback ${ }^{[32]}$ estimated the turnover rate $\left(t_{1 / 2}\right)$ of synaptic nAChRs in innervated diaphragm muscle was approximately 16 days, while that in denervated muscle was approximately 4 days. The functional importance of the increased turnover of nAChRs in denervated muscle is not 
clear, but the difference in turnover rate appears to result at least in part from a switch from expression of the $\varepsilon$ subunit, which expressed in innervated adult muscle, to that of the $\gamma$ subunit, which is normally expressed in embryonic mus$\mathrm{cle}^{[33]}$. This, then, appears to be a case in which transcriptional regulation leads to changes in trafficking properties.

Endocytosis of nAChRs on muscle also appears to play a role in the formation of neuromuscular junctions during development. In a study of mice that lacked choline acetyltransferase, the synthetic enzyme for ACh, Misgeld et al ${ }^{[34]}$ concluded that cholinergic stimulation during development disperses AChR clusters, at least in part by increased internalization of nAChRs. This was consistent with the earlier finding that agonists promote the internalization of nAChRs on myotubes in culture ${ }^{[35]}$.

In studies using repeated imaging of identified neuromuscular junctions in living adult mice, the half-time of synaptic AChRs was estimated to be approximately 14 days ${ }^{[36]}$. Blockade of neurotransmission rapidly (within $2 \mathrm{~h}$ ) decreased the half-time 25 -fold, to less than 1 day. The decrease in halftime appeared to reflect a combination of increased lateral dispersal of synaptic nAChRs and increased internalization. More recent studies using repeated in vivo imaging have provided evidence that $\mathrm{nAChRs}$ that are internalized at neuromuscular junctions can be recycled to the cell surface and reincorporated at the synapse ${ }^{[37]}$; this recycling apparently requires synaptic activity ${ }^{[37]}$. It is interesting to note that recycled nAChRs are subsequently removed from the synapse more quickly than newly inserted nAChRs that were not (yet) recycled ${ }^{[37]}$, suggesting that recycled receptors are somehow tagged or in some way differ from "naive" receptors in their interactions with other proteins.

Proper recycling of nAChRs to the synapse may require the activity of a protein tyrosine phosphatas $\mathrm{e}^{[37]}$. The latter finding appears to be consistent with earlier biochemical studies. Qu et a ${ }^{[38]}$ found evidence that denervation of skeletal muscle, which increases the turnover rate of nAChRs (above), leads to a decrease in tyrosine phosphorylation of nAChRs, and Sava et al ${ }^{[39]}$ concluded that a protein tyrosine phosphatase can promote the turnover of nAChRs in muscle. $\mathrm{Xu}$ and Salpeter ${ }^{[40]}$ found that activation of the serine/threonine protein kinase PKA also slows the turnover of nAChRs in denervated muscle.

Recent work has begun to elucidate the cellular/molecular mechanisms involved in the endocytosis of nAChRs, and early results suggest that it occurs through an unusual cellular pathway. The endocytosis of $\mathrm{nAChRs}$ can be caused by agonists ${ }^{[35]}$, by the antagonist $\alpha$-bungarotoxin ${ }^{[36]}$, or by cross-linking anti-nAChR antibodies. Kumari et al ${ }^{[41]}$, using such treatments as tools to trigger endocytosis, found that the endocytosis of $\mathrm{nAChRs}$ proceeds even in the absence of functional clathrin, caveolin, or dynamin, but that it requires the polymerization of actin, apparently through activation of Rac-1.

Proteins called neuregulins (NRGs), members of the EGF family, can alter the expression of nAChRs in muscle cells and neurons ${ }^{[42-44]}$. While NRGs can alter the transcription of nAChR subunit genes, recent studies indicate that NRGs can modulate the trafficking of nAChRs, as well. Chang and Fischbach ${ }^{[45]}$ found that NRG1 $\beta$ rapidly decreases fast responses to ACh, fast synaptic EPSCs, and cell-surface binding of $\alpha$-bungarotoxin in hippocampal neurons, suggesting that NRG1 $\beta$ triggers the rapid internalization of $\alpha 7$-nAChRs from the surface of these neurons. The effect was blocked by an inhibitor of the ErbB tyrosine kinase, which suggests that protein tyrosine phosphorylation plays a role in the process. The effect of NRG1 $\beta$ was also blocked by agents that perturb the actin cytoskeleton, which is consistent with the conclusion of Kumari et a ${ }^{[41]}$ (see above) concerning the cellular machinery for the endocytosis of nAChRs in muscle.

Sphingolipids appear to play a role in the trafficking of nAChRs in the biosynthetic pathway, as mentioned above. Interestingly, membrane cholesterol appears to affect the trafficking of nAChRs, but primarily through an effect on the endocytic pathway: depletion of cholesterol reduces the number of nAChRs on the cell surface by accelerating receptor endocytosis ${ }^{[46]}$.

\section{Trafficking of nAChRs in disease}

Trafficking of nAChRs may play a role in disease. Its role in one disease, myasthenia gravis (MG), is well established. MG is an autoimmune disease in which patients develop antibodies against nAChRs. Binding of these antibodies to nAChRs on the surface of muscle cells triggers internalization and degradation of nAChRs. Because the internalized and degraded receptors are not replaced via increased synthesis of new nAChRs, there is an overall decrease in the number of $\mathrm{nAChRs}$ at neuromuscular junctions, with a resulting loss of synaptic efficacy. Kuncl et al ${ }^{[47]}$ suggested reducing the endocytosis of nAChRs in muscle as a possible therapeutic approach for MG, but I am not aware of attempts to pursue that.

Defects in the trafficking of nAChRs in neurons may underlie additional diseases. A striking feature of Alzheimer's disease $(\mathrm{AD})$ is the selective loss of nAChRs, especially in the hippocampus and neocortex ${ }^{[48-51]}$. The cause of this 
loss is not clear, but it appears to result from a posttranslational effect ${ }^{[52]}$. The selective, possibly posttranslational loss of nAChRs could result from an $A \beta$-induced internalization of nAChRs, as hypothesized by Nagele et a ${ }^{[53]}$. Finally, Severance and Yolken ${ }^{[54]}$ have hypothesized that $\mathrm{nAChR}$ trafficking problems are involved in bipolar disorder. We can hope that additional studies will provide deeper insights into the possible role of $\mathrm{nAChR}$ trafficking in human disease.

\section{Conclusion}

1) Cellular trafficking events that could cause rapid synaptic plasticity like that observed for the trafficking of ionotropic glutamate receptors seem most likely to occur on CNS neurons, but as yet have not been clearly demonstrated.

2) Assembly and release from the ER appears to be a key regulatory point in cells, and one that may play an important role in the effects of chronic nicotine use; the UPS may play a central role in controlling ER release.

3) Several other molecules and mechanisms that can regulate $\mathrm{nAChR}$ trafficking have been identified (in addition to ER release); it is important to determine the extent to which these molecules and mechanisms account for the phenomena that have been observed that may involve trafficking effects.

4) Looking back on this time, future authors may conclude that in 2009 we were at the dawn of a golden age of progress in understanding the physiological importance and molecular mechanisms of nAChR trafficking.

\section{Acknowledgements}

The author's work was supported by American Health Assistance Foundation (№ A2006-236).

\section{References}

1 Greger IH, Esteban JA. AMPA receptor biogenesis and trafficking. Curr Opin Neurobiol 2007; 17: 289-97.

2 Lau CG, Zukin RS. NMDA receptor trafficking in synaptic plasticity and neuropsychiatric disorders. Nat Rev Neurosci 2007; 8: 413-26.

3 Shepherd JD, Huganir RL. The cell biology of synaptic plasticity: AMPA receptor trafficking. Annu Rev Cell Dev Biol 2007; 23: 613-43.

4 Huh KH, Fuhrer C. Clustering of nicotinic acetylcholine receptors: from the neuromuscular junction to interneuronal synapses. Mol Neurobiol 2002; 25: 79-112.

5 Kummer TT, Misgeld T, Sanes JR. Assembly of the postsynaptic membrane at the neuromuscular junction: paradigm lost. Curr Opin Neurobiol 2006; 16: 74-82.
6 Bruneau EG, Akaaboune M. Running to stand still. Ionotropic receptor dynamics at central and peripheral synapses. Mol Neurobiol 2006; 34: 137-51.

7 Hampton RY. ER-associated degradation in protein quality control and cellular regulation. Curr Opin Cell Biol 2002; 14: 476-82.

8 Wang JM, Zhang L, Yao Y, Viroonchatapan N, Rothe E, Wang ZZ. A transmembrane motif governs the surface trafficking of nicotinic acetylcholine receptors. Nat Neurosci 2002; 5: 963-70.

9 Keller SH, Lindstrom J, Ellisman M, Taylor P. Adjacent basic amino acid residues recognized by the COP I complex and ubiquitination govern endoplasmic reticulum to cell surface trafficking of the nicotinic acetylcholine receptor $\alpha$-subunit. J Biol Chem 2001; 276: 18384-91.

10 Ren XQ, Cheng SB, Treuil MW, Mukherjee J, Rao J, Braunewell $\mathrm{KH}$, et al. Structural determinants of $\alpha 4 \beta 2$ nicotinic acetylcholine receptor trafficking. J Neurosci 2005; 25: 6676-86.

11 Christianson JC, Green WN. Regulation of nicotinic receptor expression by the ubiquitin-proteasome system. EMBO J 2004; 23: 4156-65.

12 Corringer JP, Sallette J, Changeux JP. Nicotine enhances intracellular nicotinic receptor maturation: A novel mechanism of neural plasticity? J Physiol (Paris) 2006; 99: 162-71.

13 Rezvani K, Teng Y, Shim D, De Biasi M. Nicotine regulates multiple synaptic proteins by inhibiting proteasomal activity. J Neurosci 2007; 27: 10508-19.

14 Marchand S, Devillers-Thiery A, Pons S, Changeux JP, Cartaud J. Rapsyn escorts the nicotinic acetylcholine receptor along the exocytic pathway via association with lipid rafts. J Neurosci 2002; 22: 8891-901.

15 Sobel A, Weber M, Changeux JP. Large-scale purification of the acetylcholine-receptor protein in its membrane-bound and detergent-extracted forms from Torpedo marmorata electric organ. Eur J Biochem 1977; 80: 215-24.

16 Han H, Yang SH, Phillips WD. Overexpression of rapsyn modifies the intracellular trafficking of acetylcholine receptors. J Neurosci Res 2000; 60: 155-63.

17 Eimer S, Gottschalk A, Hengartner M, Horvitz HR, Richmond J, Schafer WR, et al. Regulation of nicotinic receptor trafficking by the transmembrane Golgi protein UNC-50. EMBO J 2007; 26: 4313-23.

18 Lin L, Jeanclos EM, Treuil M, Braunewell KH, Gundelfinger ED, Anand R. The calcium sensor protein visinin-like protein-1 modulates the surface expression and agonist sensitivity of the $\alpha 4 \beta 2$ nicotinic acetylcholine receptor. J Biol Chem 2002; 277 : 41872-8.

19 Coussen F, Perrais D, Jaskolski F, Sachidhanandam S, Normand E, Bockaert J, et al. Co-assembly of two GluR6 kainate receptor splice variants within a functional protein complex. Neuron 2005; 47: 555-66.

20 Zhao CJ, Noack C, Brackmann M, Gloveli T, Maelicke A, Heinemann $\mathrm{U}$, et al. Neuronal $\mathrm{Ca}^{2+}$ sensor VILIP-1 leads to the upregulation of functional $\alpha 4 \beta 2$ nicotinic acetylcholine receptors in hippocampal neurons. Mol Cell Neurosci 2009; 40: 280-92.

21 Nashmi R, Dickinson ME, McKinney S, Jareb M, Labarca C, Fraser $\mathrm{SE}$, et al. Assembly of $\alpha 4 \beta 2$ nicotinic acetylcholine receptors assessed with functional fluorescently labeled subunits: effects of localization, trafficking, and nicotine-induced upregulation in clonal mammalian cells and in cultured midbrain neurons. J 
Neurosci 2003; 23: 11554-67.

22 Mrowiec T, Schwappach B. 14-3-3 proteins in membrane protein transport. Biol Chem 2006; 387: 1227-36.

23 Bermudez I, Moroni M. Phosphorylation and function of $\alpha 4 \beta 2$ receptor. J Mol Neurosci 2006; 30: 97-8.

24 Rosenberg MM, Yang F, Giovanni M, Mohn JL, Temburni MK, Jacob MH. Adenomatous polyposis coli plays a key role, in vivo, in coordinating assembly of the neuronal nicotinic postsynaptic complex. Mol Cell Neurosci 2008; 38: 138-52.

25 Castillo M, Mulet J, Gutierrez LM, Ortiz JA, Castelan F, Gerber $\mathrm{S}$, et al. Dual role of the RIC-3 protein in trafficking of serotonin and nicotinic acetylcholine receptors. J Biol Chem 2005; 280: 27062-8.

26 Cho C-H, Song W, Leitzell K, Teo E, Meleth AD, Quick MW, et al. Rapid upregulation of $\alpha 7$ nicotinic acetylcholine receptors by tyrosine dephosphorylation. J Neurosci 2005; 25: 3712-23.

27 Baier CJ, Barrantes FJ. Sphingolipids are necessary for nicotinic acetylcholine receptor export in the early secretory pathway. J Neurochem 2007; 101: 1072-84.

28 Gentry CL, Lukas RJ. Regulation of nicotinic acetylcholine receptor numbers and function by chronic nicotine exposure. Curr Drug Targets CNS Neurol Disord 2002; 1: 359-85.

29 Darsow T, Booker TK, Pina-Crespo JC, Heinemann SF. Exocytic trafficking is required for nicotine-induced up-regulation of $\alpha 4 \beta 2$ nicotinic acetylcholine receptors. J Biol Chem 2005; 280: 1831120.

30 Vallejo YF, Buisson B, Bertrand D, Green WN. Chronic nicotine exposure upregulates nicotinic receptors by a novel mechanism. J Neurosci 2005; 25: 5563-72.

31 Merlie JP, Isenberg KE, Russell SD, Sanes JR. Denervation supersensitivity in skeletal muscle: analysis with a cloned cDNA probe. J Cell Biol 1984; 99: 332-5.

32 Bevan S, Steinbach JH. Denervation increases the degradation rate if acetylcholine receptors at end-plates in vivo and in vitro. J Physiol 1983; 336: 159-77.

33 Sala C, O’Malley J, Xu R, Fumagalli G, Salpeter MM. $\varepsilon$ Subunitcontaining acetylcholine receptors in myotubes belong to the slowly degrading population. J Neurosci 1997; 17: 8937-44.

34 Misgeld T, Kummer T, Lichtman JW, Sanes JR. Agrin promotes synaptic differentiation by counteracting an inhibitory effect of neurotransmitter. Proc Natl Acad Sci USA 2005; 102: 11088-93.

35 St John PA, Gordon H. Agonists cause endocytosis of nicotinic acetylcholine receptors on cultured myotubes. J Neurobiol 2001; 49: 212-23.

36 Akaaboune M, Culican SM, Turney SG, Lichtman JW. Rapid and recersible effects of activity on acetylcholine receptor density at the neuromuscular junction in vivo. Science 1999; 286: 503-7.

37 Bruneau EG, Akaaboune M. The dynamics of recycled acetylcholine receptors at the neuromuscular junction in vivo. Development 2006; 133: 4485-93.

$38 \mathrm{Qu} \mathrm{Z}$, Moritz E, Huganir RL. Regulation of tyrosine phosphorylation of the nicotinic acetylcholine receptor at the rat neuromuscular junction. Neuron 1990; 2: 367-78.

39 Sava A, Barisone I, Di Mauro D, Fumagalli G, Sala C. Modulation of nicotinic acetylcholine receptor turnover by tyrosine phos- phorylation in rat myotubes. Neurosci Lett 2001; 313: 37-40.

$40 \mathrm{Xu} \mathrm{R}$, Salpeter MM. Protein kinase A regulates the degradation rate of Rs acetylcholine receptors. J Cell Physiol 1995; 165: 30-9.

41 Kumari S, Borroni V, Chaudhry A, Chandra B, Massol R, Mayor $S$, et al. Nicotinic acetylcholine receptor is internalized via a Racdependent, dynamin-independent endocytic pathway. J Cell Biol 2008; 181: 1179-93.

42 Yang X, Kuo Y, Devay P, Yu C, Role L. A cysteine-rich isoform of neuregulin controls the level of expression of neuronal nicotinic receptor channels during synaptogenesis. Neuron 1998; 20: 25570.

43 Liu Y, Ford B, Mann MA, Fischbach GD. Neuregulins increase alpha7 nicotinic acetylcholine receptors and enhance excitatory synaptic transmission in GABAergic interneurons of the hippocampus. J Neurosci 2001; 21: 5660-9.

44 Kawai H, Zago W, Berg DK. Nicotinic alpha 7 receptor clusters on hippocampal GABAergic neurons: regulation by synaptic activity and neurotrophins. J Neurosci 2002; 22: 7903-12.

45 Chang Q Fischbach GD. An acute effect of neuregulin $1 \beta$ to suppress $\alpha 7$-containing nicotinic acetylcholine receptors in hippocampal interneurons. J Neurosci 2006; 26: 11295-303.

46 Borroni V, Baier CJ, Land T, Bonini I, White MM, Garbus I, et al. Cholesterol depletion activates rapid internalization of submicronsized acetylcholine receptor domains at the cell membrane. Mol Membr Biol 2007; 24:1-15.

47 Kuncl RW, Wittstein I, Adams RN, Wiggins WW, Avila O, Pestronk A, et al. A novel therapy for myasthenia gravis by reducing the endocytosis of acetylcholine receptors. Ann N Y Acad Sci 1993; 681: 298-302.

48 Court J, Martin-Ruiz C, Piggott M, Spurden D, Griffiths M, Perry E. Nicotinic receptor abnormalities in Alzheimer's disease. Biol Psychiatry 2001; 49: 175-84.

49 Marutle A, Warpman U, Bogdanovic N, Lannfelt L, Nordberg A. Neuronal nicotinic receptor deficits in Alzheimer patients with the Swedish amyloid precursor protein 670/671 mutation. J Neurochem 1999; 72: 1161-9.

50 Nordberg A. Nicotinic receptor abnormalities of Alzheimer's disease: therapeutic implications. Biol Psychiatry 2001; 49: 20010.

51 Perry EK, Morris CM, Court JA, Cheng A, Fairbairn AF, McKeith IG, et al. Alterations in nicotine binding sites in Parkinson's disease, Lewy body dementia and Alzheimer's disease: possible index of early neuropathology. Neuroscience 1995; 64: 385-95.

52 Hellstrom-Lindahl E, Mousavi M, Zhang X, Ravid R, Nordberg A. Regional distribution of nicotinic receptor subunit nRNAs in human brain: comparison between Alzheimer and normal brain. Brain Res Mol Brain Res 1999; 66: 94-103.

53 Nagele RG, D’Andrea MR, Anderson WJ, Wang HY. Intracellular accumulation of $\beta$-amyloid1-42 in neurons is facilitated by the $\alpha 7$ nicotinic acetylcholine receptor in Alzheimer's disease. Neuroscience 2002; 110: 199-211.

54 Severance EG, Yolken RH. Lack of Ric-3 congruence with $\beta 2$ subunit-containing nicotinic acetylcholine receptors in bipolar disorder. Neuroscince 2007; 148: 454-60. 